\title{
AN ANALYSIS FOR PARALLEL WIND SIMULATION SPEEDUP USING OPENFOAM *
}

\author{
NEKI FRASHERI ${ }^{\dagger}$ AND EMANOUIL ATANASSOV $\ddagger$
}

\begin{abstract}
An analysis of speedup for parallel execution of OpenFOAM software for wind simulation over rugged terrain is presented in the paper. Runtime speedup is analyzed using small and medium resolution DEM models for icoFoam and pisoFoam solvers, the latter due to consideration of turbulence, running in the parallel system Avitohol of Institute of Information and Communication Technologies of the Bulgarian Academy of Sciences. The results gave a clearer view about the possibility to run in reasonable time medium and high resolution models in regional scale, while indicating the weight of turbulence calculations for computing runtime requirements.
\end{abstract}

Key words: OpenFOAM; wind simulation; HPC scalability

AMS subject classifications. 68U20, 68W10, 68W40

1. Introduction. The H2020 project VI-SEEM aims at creating a unique Virtual Research Environment (VRE) in Southeast Europe and the Eastern Mediterranean (SEEM), with special focus on the scientific communities of Life Sciences, Climatology and Digital Cultural Heritage [https://vi-seem.eu/]. One of the tasks in the project was to realize in VI-SEEM VRE the wind simulation over rugged terrain in regional scales, important for environmental studies and green energy production, which had to be undertaken by Polytechnic University of Tirana (UPT), Albania.

Involved for the first time in such simulations at UPT, we selected for this purpose the OpenFOAM software [https://www.openfoam.com/], which is one of available open source packages to solve Navier-Stokes equations for fluid dynamics, applicable for air flows.

Tanasescu has presented OpenFOAM as a leading Open Source CFD (Computational Fluid Dynamics) having qualities of accessibility, transparency, customization, and extensible [1]. The package offers a multitude of solvers, parallelized with MPI, for different Navier-Stokes problems.

A number of factors that impact the volume of requested computing capacities were identified during the literature review, related with the scalability dependence from the nature of concrete models, model size, interprocess communication, etc. In particular, air flow around sails solutions are presented in [2] by Lombardi et al, and the impact of data interchange between cores is evaluated. Ravelli et al analyzed air flows in turbines [3]. Lysenko et al presented several cases of turbulent flows in [4], considering both weak and strong scalability for the OpenFOAM. Rivera et al studied large eddy flows and related scalability [5].

Dagna and Hertzer studied the scalability of OpenFOAM with hybrid MPI and OpenMP parallelization with up to 4096 cores in BlueGene/Q system [6]. Lysenko et al simulated aerodynamic sound from a circular cylinder using up to 256 cores with an average efficiency of $70 \%$ [7]. Karasek et al compared different benchmarks for evaluation of OpenFOAM performance using up to 1024 cores [8].

Kornyei studied the speedup and scalability of simulating combustible gas flows with up to 256 cores in [9]. Ponweiser et al presented fluid-structure simulations for aircraft design in [10], dealing with memory limitations for limited number of 128-256 cores. Sidlof and Ridky studied the scalability of simulated flows past airfoil in [11], experiencing $50 \%$ speedup reduction when over 36 cores were used.

Atmospheric simulations were done by Flores et al for turbulent flows over complex urban geometry [12]. Vermier et al analysed the atmospheric boundary layer in complex terrain [13]. Garcia et al studied the buoyancy effect of temperature over terrain, using SRTM Digital Elevation Model and Landsat infrared imagery for the ground temperature [14].

Garcia and Boulanger simulated the altitude winds over mount Saint Helens using SRTM Digital Elevation Model [15]. Hardin offered a solution how to build OpenFOAM terrain mesh using Digital Elevation Models

\footnotetext{
*The work presented in this paper has been supported by EU under the H2020 programme Grant agreement no. 675121 VRE for regional Interdisciplinary communities in Southeast Europe and the Eastern Mediterranean (VI-SEEM).

${ }^{\dagger}$ Faculty of Information Technology, Polytechnic University of Tirana Tirana, Albania (nfrasheri@fti.edu.al).

$\ddagger$ Institute of Information and Communication Technologies, Bulgarian Academy Of Sciences, Sofia, Bulgaria (emanouil@parallel.bas.bg).
} 
from GRAS GIS system [16]. Tapia made a synthesis of theoretical issues of OpenFOAM with cases of wind farms and simple hill terrain [17].

Most of the reviewed literature presented for concrete problems some runtime analysis, but without details on memory usage, which resulted to be critical in our case and confirmed the remark of Culpo that the size of problems that can be handled on a HPC cluster lies beyond the limitations imposed by smaller in-house clusters [18].

Our study was focused on the evaluation of OpenFOAM scalability when it runs for 3D regional wind simulations in HPC systems in our disposal. Simulations were planned for the geographical region that includes Albania, two thirds of which is mountainous. In order to include rugged terrain in OpenFOAM data, we used the Digital Elevation Model (DEM) fragment of mountainous area including Albania from the NASA Shuttle Radar Topography Model (SRTM) data obtained from USGS archive [https://earthexplorer.usgs.gov/]. The selection of SRTM DEM was based on the facts that it can be obtained freely from the Internet and we had previous experiences with its usage.

The models were designed to take into account air turbulence, which requires iterative solution of equations for a sequence of time intervals and periodical storage of related temporal results in disk space. While increasing the model resolution through decreasing of spatial mesh steps, another problem emerged related with the balance between spatial and temporal discretization steps [19], leading to the need of decreasing time steps proportionally with spatial steps, and increasing the number of iterations in order to keep the same time span.

First experiments were carried out in local workstations and the small multiprocessor system of UPT, obtaining a first evaluation of computing capacities, as a preparatory phase for running it in the VI-SEEM VRE [20], [21]. In the actual paper results from new experiments in Avitohol are presented.

2. Experimental Setup. Atmospheric winds can be simulated solving Navier-Stokes equations applied for incompressible laminar and turbulent flows in spatial 3D volumes. Equations include the temporal dimension as well, especially necessary for turbulence problems. Software OpenFOAM solves Navier-Stokes equations using finite volume methods based on digitized spatial 3D mesh, through a suite of iterations in temporal dimension. In order to include the effect of buildings and mountains in air flows, 3D mesh generated for a rectangular volume should be deformed following a digital elevation model of the relief.

Mountainous ranges in Western Balkans are cut by a network of narrow deep valleys, which impact the air flows due to meteorological conditions. Valleys less than $1 \mathrm{~km}$ wide require wind simulation models of high resolution. NASA SRTM DEM with 3 arcsec per pixel, which corresponds with a rectangular metric resolution of 100x100m in equator and 70x100m in latitudes 40 degrees (meridians are nearer each other farther from equator), and it would be suitable for wind simulations in narrow valleys. We selected the DEM section covering Albania with the highest resolution available 3 arcsec per pixel with size 3600x4800 pixel (Fig. 2.1.a), defined with corner coordinates in degrees, minutes and seconds:

Upper Left (18d59'58.50”E, 43d 0' 1.50”N)

Lower Left (18d59'58.50"E, 38d59'58.50”N)

Upper Right (22d 0' 1.50 "E, 43d 0' 1.50” N)

Lower Right (22d 0' 1.50”E, 38d59'58.50”N)

The 3D model size of lower atmosphere volume in kilometers was 270x480x10, where the variation of arcsec metric distances by latitude was considered. Our experiments were based on several models of the same volume with different resolutions in the range from DEM 36x48 pixels up to 500x667 pixels obtained reducing proportionally the Fig. 2.1.a image, with a spatial meridian step varying respectively from $10 \mathrm{~km}$ to $720 \mathrm{~m}$.

The characteristics of used model resolutions are presented in Table 2.1, where respective numbers of digitized finite volume elements in three spatial dimensions (X, Y, and $\mathrm{Z}$ ), their total number, and requested virtual memory (RAM) for each case are presented. The factor dedicated for each of models, proportional with related one-dimensional size in pixels, is used to simplify the graphical presentation of results. It was not possible to run models with higher resolutions, for which the extrapolation was used to evaluate requested computing capacities.

The Reynolds number for the air was taken 1e-5, situated in the file transportProperties. Boundary conditions were defined in files $0 / p$ and $0 / U$, applied for boundary faces of 3D rectangle in the file blockMeshDict as 

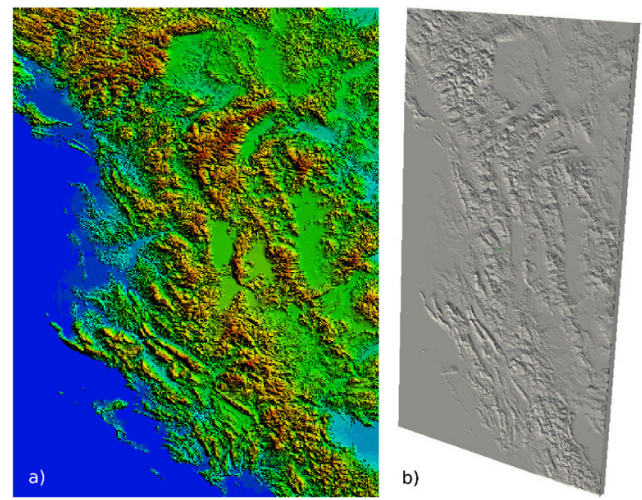

FIG. 2.1. a) $3600 x 4800$ DEM image for Albania and surrounding area; b) bottom face of $3 D$ volume deformed based on DEM.

TABLE 2.1

Model sizes used for experiments

\begin{tabular}{|c|c|c|c|c|c|}
\hline Factor & X pixels & Y pixels & Z pixels & Elements & RAM KB \\
\hline \hline 10 & 36 & 48 & 10 & $1.73 \mathrm{E}+04$ & $1.20 \mathrm{E}+05$ \\
\hline 43 & 154 & 206 & 43 & $1.36 \mathrm{E}+06$ & $1.25 \mathrm{E}+06$ \\
\hline 60 & 216 & 288 & 60 & $3.73 \mathrm{E}+06$ & $3.07 \mathrm{E}+06$ \\
\hline 100 & 360 & 480 & 100 & $1.73 \mathrm{E}+07$ & $1.31 \mathrm{E}+07$ \\
\hline 139 & 500 & 667 & 139 & $4.64 \mathrm{E}+07$ & $3.34 \mathrm{E}+07$ \\
\hline 1000 & 3600 & 4800 & 1000 & $1.73 \mathrm{E}+10$ & $1.43 \mathrm{E}+09$ \\
\hline
\end{tabular}

follows (orientation of front-end and left-right faces were defined following the direction of the wind north-south):

- front end vertical faces: fixed walls with uniform values +1 and -1 and zero gradients

- left and right vertical faces: fixed zero gradient walls

- top horizontal face (upper atmosphere): fixed zero gradient wall

- bottom horizontal face (ground surface): fixed zero values wall

The model meshes were generated with the OpenFOAM module blockMesh. Before running OpenFOAM solvers, the generated spatial coordinates of mesh nodes situated in the file ./constant/polyMesh/points were modified to include the relief, modifying the altitudes of nodes starting from the bottom of mesh (ground surface) and decreasing linearly to zero until the top.

Similar with other files used by OpenFOAM, the file points contains in editable format ASCII the data: a header and the suite of coordinates $(\mathrm{x}, \mathrm{y}, \mathrm{z})$ of mesh nodes. DEM files were downloaded from USGS repository in binary format. GDAL software tools [http://www.gdal.org/] were used to process binary DEM data, using gdalinfo to obtain related metadata, and gdal_translate to convert data into Surfer ASCII grid format [http://www.tifton.uga.edu/sewrl/flownet/flownet.htm]. The already known and editable grid text format contains the header with number of pixels and the $2 \mathrm{D}$ array of heights in meters, which were used to modify vertical coordinates in points file using an in-house written software.

Splitting of input data for parallel processing with the solver module and recombination of partial results were done with modules decmposePar dhe reconstructPar. Used OpenFOAM MPI prallelized solvers were icoFoam for incompressible laminar flows and pisooFoam for turbulent flows.

The execution of OpenFOAM suite in parallel requires running of four modules: the mesh generator blockMesh (necessary even for simple modification of boundary conditions), followed by the decomposePar module, the solver (icoFoam and pisoFoam in our case), terminating with reconstructPar module.

The OpenFOAM solution gives the distribution of scalar potential and vectorial velocity fields, for the latter magnitude and three vector components are given for axes $\mathrm{X}$ (west to east), $\mathrm{Y}$ (south to north), and Z (bottom-up). 

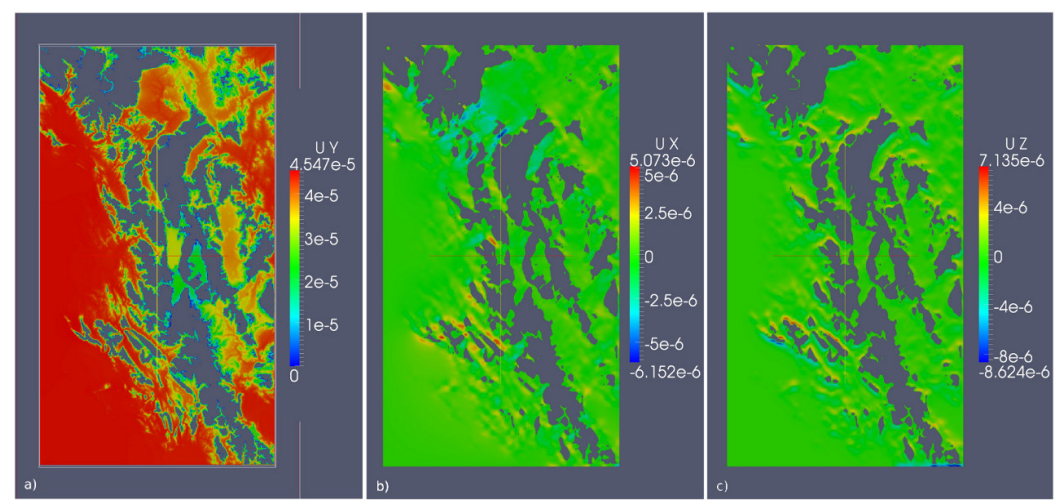

FIG. 3.1. a) North-south wind flow in altitude 1000m; b) induced west-east wind flow in altitude 1000m; c) induced vertical wind flow in altitude $1000 \mathrm{~m}$.

Recent experiments were carried out running OpenFOAM solver for 10,000 time steps of length 0.001 second, storing results in external storage at the end of each second for duration of 10 seconds. The same was done for all model sizes, taking into account the time step length required for higher resolution models. Use of small spatial steps with long time steps in previous experiments has led to miss-balance between spatial and temporal discretization, increasing courant numbers greater that one (the case when fluid particles jump over one mesh cell while moving from one time instance to the next one), and the divergence of iterative process [19].

The used computer system was the supercomputer Avitohol, with 150 servers with dual Intel Xeon E52650v2 8C @2.6GHz running Scientific Linux 6 and Intel compilers, in the Institute of Information and Communication Technologies, Bulgarian Academy of Sciences (IICT-BAS).

3. Preliminary Simulation Results. The used SRTM DEM data (Fig. 2.1) cover territories of Albania and small parts of surrounding countries. The area is characterized by a group of high Alps Mountains in North, and ranges of mountains that extend in direction north-north-west to south-south-east cut by narrow river valleys flowing from east to west. In the western part of this area there is the PreAdriatic Depression, lowlands bordered by Adriatic Sea.

Experiments presented here were done with boundary conditions for a constant regional wind flowing north to south, using potentials +1 and -1 in respective northern and southern faces of the $3 \mathrm{D}$ volume. We used zero conditions for the bottom ground face, and gradient zero on the rest of faces (east, west and top).

In the Fig. 3.1 there are presented the distribution of magnitude and components $\mathrm{X}$ and $\mathrm{Z}$ of the velocity field in a plan at altitude $1,000 \mathrm{~m}$, where tops of mountains are shown in gray. A near to surface reduction of wind magnitude is visible around mountain peaks. Low values of velocity are characteristic for protected closed mountainous valleys (image 'a'), with a decrease of magnitude down to $25 \%$.

The effect of relief is more visible in images 'b' and 'c' presenting west - east and vertical wind flows. Due to oblique extension of mountain ranges, in valleys there is generated a component of west - east wind flow with magnitude 50\%, flowing to the east or west depending to the orientation of open valleys. In the image 'c' strong vertical wind flow component is visible in high mountain slopes facing north. This phenomenon is significant in two areas - northern boundaries of Mirdita and mountainous areas east of Vlora city.

The results show the importance of such simulations for planning of wind energy farms, and for the air transport. A more detailed analysis is necessary for such purpose, considering the complexity of relief.

4. OpenFOAM Scalability In Avitohol. New results presented in this paper consist of runtime analysis for the solver icoFoam and comparison of both runtime and disk space required by both solvers icoFoam and pisoFoam, for models which sizes are described in Table 2.1, except for the case of highest resolution for which extrapolation was used in few cases.

The volume of virtual memory resulted similar for all modules depending on the quantity of 3D mesh nodes (Fig. 4.1; while volume of external storage correlates with the virtual memory size multiplied with the number of stored temporal results (in the figure case of 100 results is presented). 


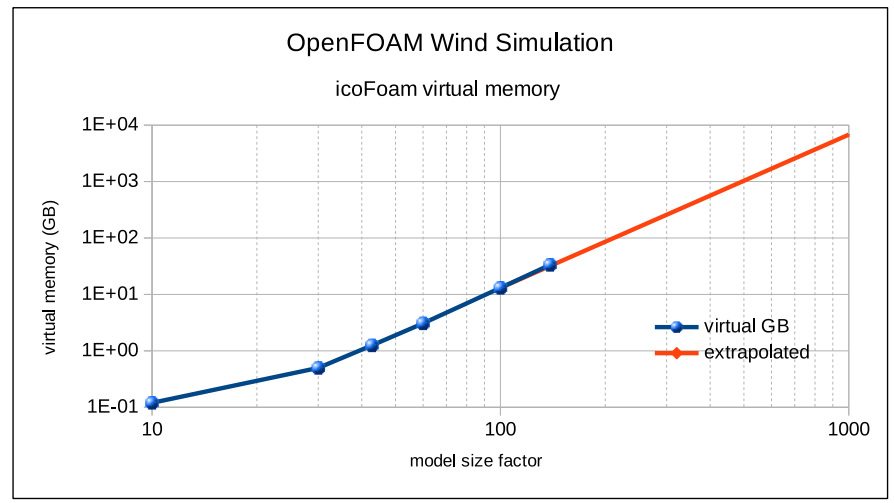

FIG. 4.1. Memory usage of OpenFOAM - single process run.

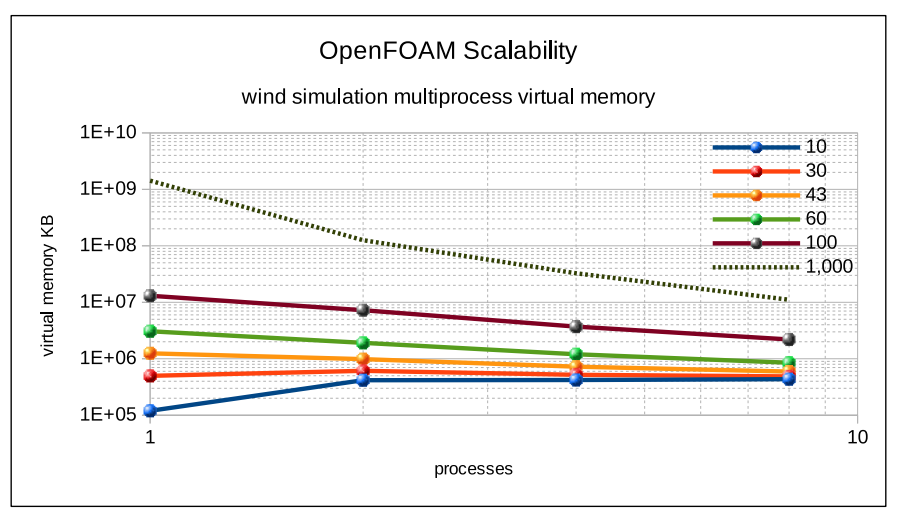

FIG. 4.2. Memory usage of OpenFOAM - multi process run.

Only the solver was possible to run in parallel, with this case due to the splitting of 3D mesh between processes, for each of them the required virtual memory is reduced converging towards around 0.5 GigaBytes per process with the increase of parallelism (Fig. 4.2).

Other pre- and post- processing modules required the total of virtual memory, decomposing and reconstructing modules duplicate the volume of data in external storage (each solver process stores its partial results and during post-processing there are stored combined files). Central memory requirements, time steps, and runtime conditioned the size of models executed in Avitohol.

Increase of time steps in order to have the requested temporal span of dynamic solutions when increasing mesh resolution was necessary to avoid the iterations divergence when spatial and temporal discretization steps were not balanced leading to the increase of courant numbers, a case of such divergence is presented in Fig. 4.3.

Runtime of different models per number of processes is shown in Fig. 4.4. For the model size 100 a trend line is calculated extrapolating the runtime for 1,000 processes.

The same runtime plotted versus the size of models is shown in Fig. 4.5. The trend line is calculated for a single process run of different models, extrapolating the runtime for single process run of the highest model size.

The speedup of parallel execution for different model sizes is given in Fig. 4.6. The lowest resolution model speedup degenerates quickly while for other models the trend seems constant for the used range 1:64 of the number of processes.

Efficiency of parallelization is given in Fig. 4.7. Even for higher resolution models the trend of reduction of efficiency is visible. The reduction trend of efficiency is expected to reach values of $50 \%$ when running models with up to 1,000 parallel processes. Further degradation of efficiency due to virtual memory requirements was not considered in these extrapolations. 


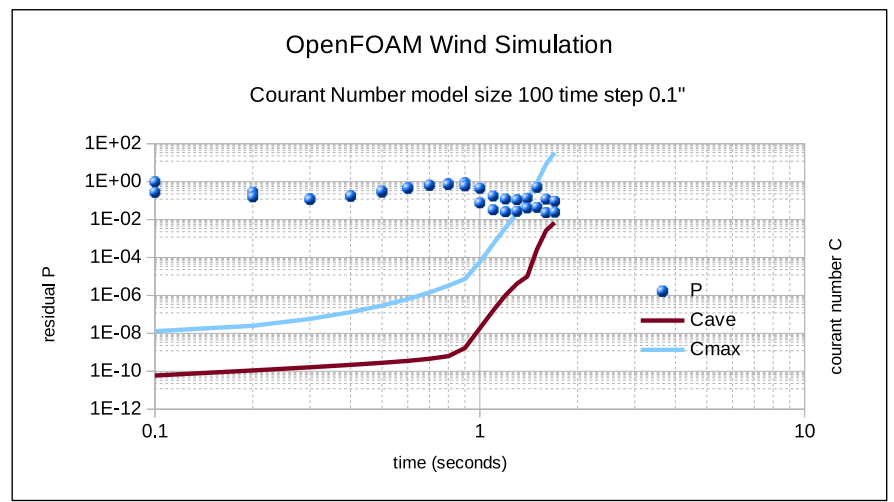

FIG. 4.3. Divergence of solution processes: error of potential field P, average Cave and maximal courant Cmax numbers for time steps 0.1 seconds.

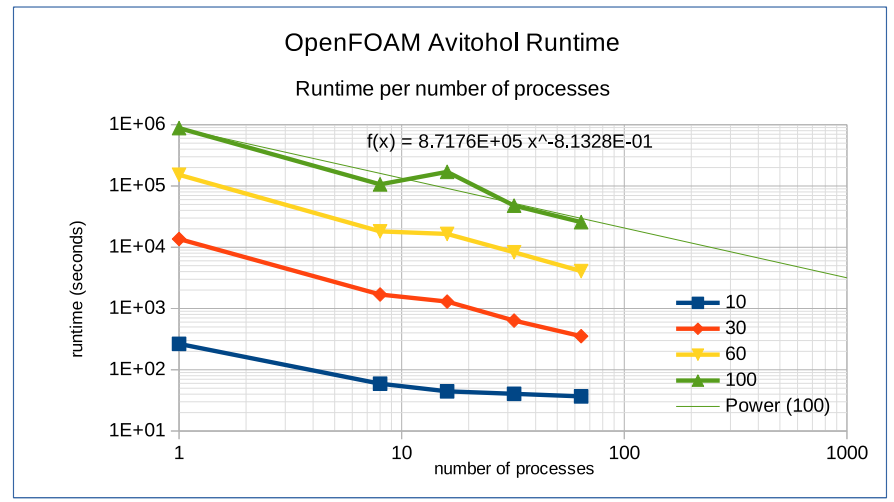

FIG. 4.4. Runtime of models per number of parallel processes.

Comparing the trends of runtime for different number of parallel processes in Fig. 4.5, it was possible to make an simple approximate evaluation and extrapolation of runtime for the highest resolution model (of factor 1,000) for up to 640 parallel processes. The runtime trends for different process numbers are parallel with the sequential run. Extrapolating the trend of 64 processes for the model factor 1,000 the approximate value $1.00 \mathrm{E}+8$ was obtained. Supposing $100 \%$ efficiency, evaluated runtime for the highest model size for 64 and 640 processes given in Table 4.1 .

Comparison of solver icoFoam and pisoFoam was done in two planes - runtime and external storage requirements. Comparison of the runtime is given in Fig. 4.8. Runtime trend in case of pisoFoam resulted at least $35 \%$ more compared with icoFoam.

Comparison of icoFoam and pisoFoam external storage requirements is given in Fig. 4.9. Requirements for both solvers are quite similar with each other, indicating that the use of pisoFoam does not require excessive extra external storage compared with icoFoam.

The runtime problem with pisoFoam is related with long time span of solution sequences necessary to indicate turbulence and eddies, compared with laminar solutions.

5. Conclusions. Usage of OpenFOAM for wind simulation models of medium resolution (spatial discretization steps $10 \mathrm{~km}-1 \mathrm{~km}$ ) over rugged mountainous regional area of Albania and surroundings was possible in the Avitohol using reasonable computational resources. Beside the runtime, another limitation was the virtual memory in levels of 10-30 GB for medium sized models, requested by preparatory modules blockMesh, decomposePar, and reconstructPar.

An optimistic extrapolation of the runtime for models with resolution $100 \mathrm{~m}$ of spatial discretization step 


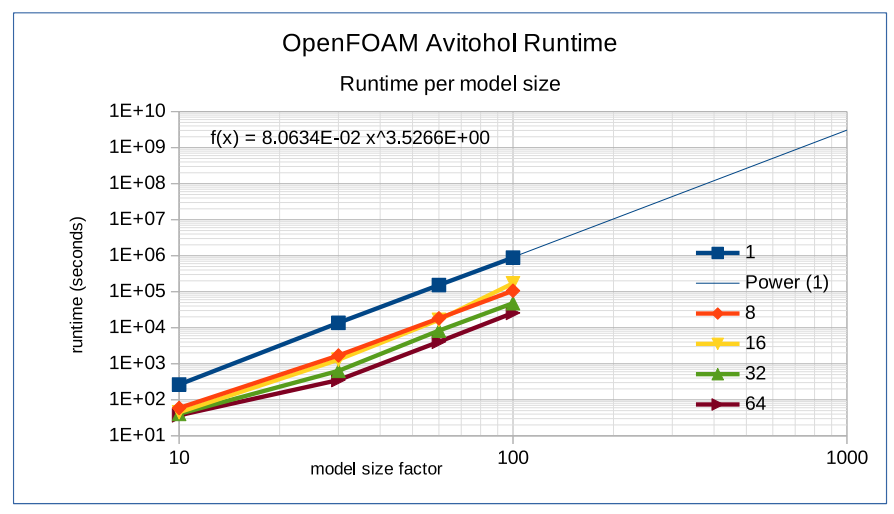

FIG. 4.5. Runtime of parallel processes per model size.

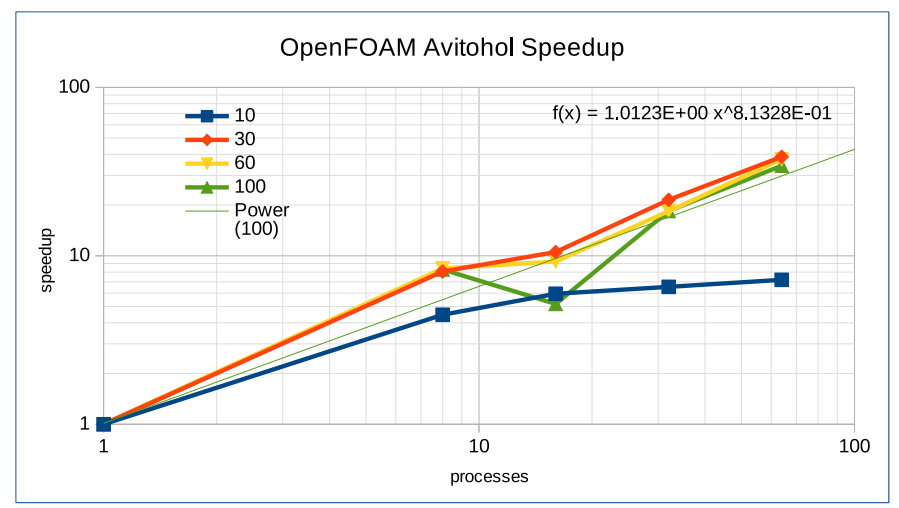

Fig. 4.6. Speedup of parallel execution in Avitohol

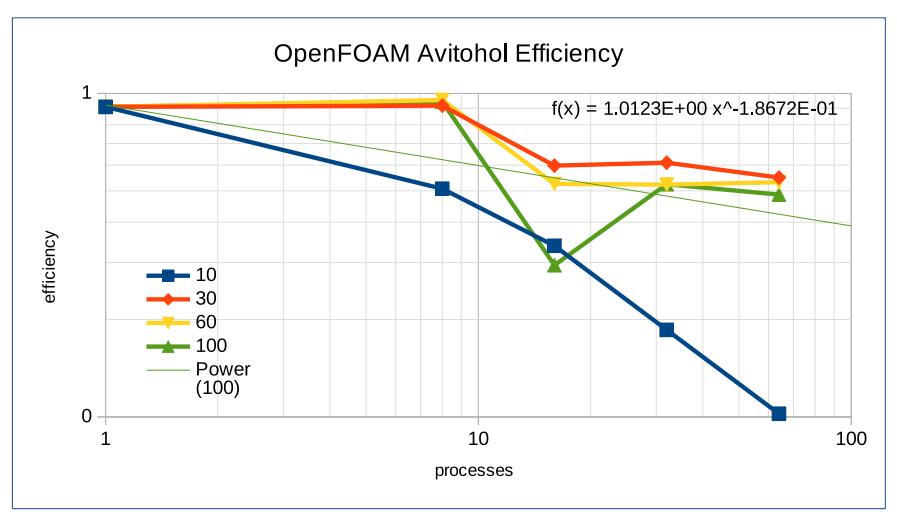

FIG. 4.7. Efficiency of parallel execution in Avitohol

TABLE 4.1

Model sizes used for experiments

\begin{tabular}{|c|c|c|c|c|c|}
\hline processes & seconds & hours & days & months & years \\
\hline \hline 64 & $1.00 \mathrm{E}+08$ & 27,778 & 1,157 & 37.95 & 3.16 \\
\hline 640 & $1.00 \mathrm{E}+07$ & 2,778 & 116 & 3.79 & 0.32 \\
\hline
\end{tabular}




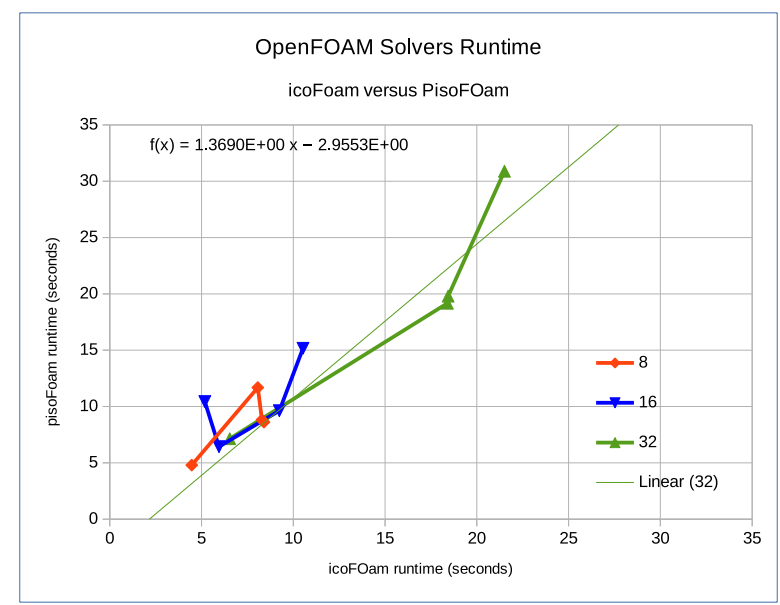

FIG. 4.8. icoFoam versus pisoFoam runtime

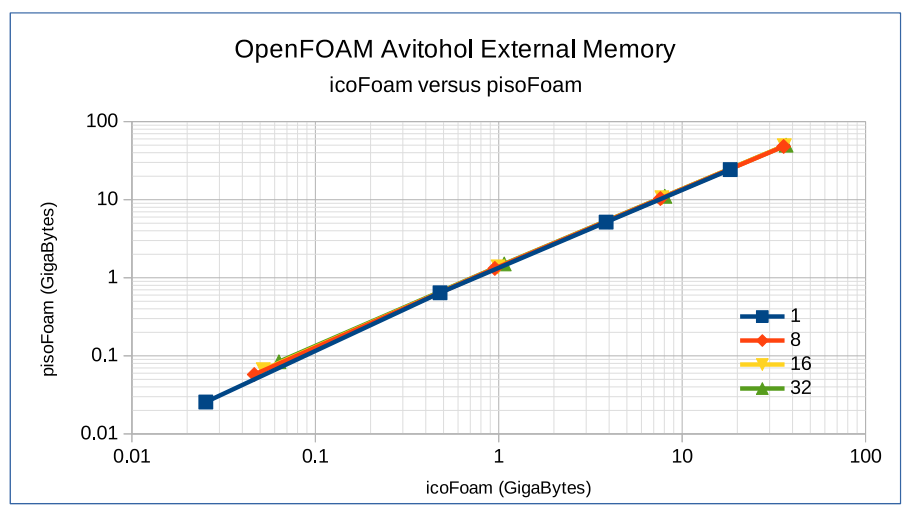

FIG. 4.9. icoFoam versus pisoFoam external storage requirements

using 640 parallel processes resulted at least 4 months, not considering limitations due to virtual memory and degeneration of efficiency from inter-process and external storage communication. Comparison of two OpenFOAM solvers, icoFoam for laminar flows and pisoFoam for turbulence flows showed that the latter requires about $35 \%$ runtime more than the former, while memory requirements are similar. It is also necessary to consider studies of turbulence and eddies would require the storage in disk of a greater number of temporal results, compared with experiment setup presented in this paper.

Acknowledgments. The research leading to these results has been co-funded by the European Commission under the H2020 Research Infrastructures contract no. 675121 (project VI-SEEM). Authors are grateful for all the help of the staff of IICT-BAS involved with the preparation of the OpenFOAM environment. Collaboration in framework of COST Action IC1305 NESUS promoted the speedup of this work.

\section{REFERENCES}

[1] C. TAnASescu, OpenFOAM and SGI designed to work together, http://docplayer.net/3118206 -Openfoam-and-sgi-designedto-work-together-christian-tanasescu-vice-president-software- engineering.html

[2] M. Lombardi, N. Parolini, A. Quarteroni, and G Rozza, Numerical simulation of sailing boats : dynamics, FSI, and shape optimization, MATHICSE Technical Report No. 03.2011, April 2011.

[3] S. Ravelli, G. Barigozzi, F. Pasqua, R. Pieri, and R. Ponzini, Numerical and experimental study for the prediction of the steady, three dimensional flow in a turbine nozzle vane cascade using OpenFOAM, in International CAE Conference 2014, Verona, Italy, October 2014, pp. 27-28. 
[4] D. Lysenko, I. ERTesvag, And K. Rian, Testing of OpenFOAM CFD code for plane turbulent bluff body flows within conventional URANS approach, in International Conference on Computational Fluid Dynamics in the Oil Gas, Metallurgical and Process Industries - CFD11, Trondheim, Norway, June 2011.

[5] O. Rivera, K. Furlinger, And D. Kranzlmuller, Investigating the scalability of OpenFOAM for the solution of transport equations and large eddy simulations, in ICA3PP11 Proceedings of the 11th International Conference on Algorithms and Architectures for Parallel Processing Volume Part II, LNCS 7017, 2011, pp. 121-130.

[6] P. DAgna AND J. HeRTZER, Evaluation of multi-threaded OpenFOAM hybridization for massively parallel architectures, Partnership for Advanced Computing in Europe (PRACE) white paper, 2017, http://www.prace-ri.eu/IMG/pdf/wp98.pdf

[7] D.A. Lysenko, I.S. ERTESVAg, And K.E.Rian, Towards simulation of far-field aerodynamic sound from a circular cylinder using OpenFOAM, Aeroacoustics, vol. 13, no. 1, 2014.

[8] T. Karasek, D. Horak, V. Hapla, A. Markopoulos, L. Riha, V. Vondrak, And T. Brzobohaty, Application of CFD and CSM open source codes for solving multiscale multiphysics problems, IT4Innovations, VSB - Technical University of Ostrava, www.prace-ri.eu

[9] L. KorNyEI, Simulation of gas flow in a combustion chamber using high performance computing hardware, in Workshop on the Occasion of the 60th Birthday of Ferenc Igloi, Budapest, Hungary, October 3, 2012.

[10] T. Ponweiser, P. Stadelmeyer, and T. Karasek, Fluid-structure simulations with OpenFOAM for aircraft designs, Partnership for Advanced Computing in Europe (PRACE) Report, www.prace-ri.eu/IMG/pdf/wp172.pdf

[11] P. SidLOF AND V. RIDKY, Scalability of the parallel CFD simulations of flow past a fluttering airfoil in OpenFOAM, in EPJ Web of Conferences 92, (2015) DOI: 10.1051/ epjconf / 20159202080.

[12] F. Flores, R. Garreaud AND R. C. MunOz, CFD simulations of turbulent buoyant atmospheric flows over complex geometry: Solver development in OpenFOAM, Elsevier Computers \& Fluids 82 (2013) 113.

[13] J. Vermeir, M. RunACres AND T. DE Troyer, CFD modelling of the boundary layer in complex terrain validated by field measurements, Proceedings of the EWEA Annual Event, 19 Apr 2012, Copenhagen.

[14] M. Garcia, P. Boulanger AND D. GiRAldo, CFD analysis effect buoyancy due terrain temperature based integrated DEM landsat infrared imagery, Ingenieria y Ciencia, ISSN 17949165, Vol. 4, no. 8, December 2008, pp. 6584

[15] M. GARcia AND P. Boulanger, Low altitude wind simulation over mount Saint Helens using NASA SRTM Digital Terrain Model, Proceedings of the Third International Symposium on 3D Data Processing, Visualization, and Transmission (3DPVT'06) 0-7695-2825-2/06, IEEE 2006.

[16] E. HARDIN, Simulating wind over terrain: how to huild an OpenFOAM case from GRASS GIS Digital Elevation Models, 2013, http://www.cybermanual.com/simulating- wind-over-terrain-how-to-build-an-openfoam-case-from-grassgis-digital-elevation-models /download.html

[17] X. P. TAPIA, Modelling of wind flow over complex terrain using OpenFoam, Masters Thesis in Energy Systems, June 2009, http://www.diva-portal.org/smash/get/diva2

[18] M. CulPo, Current bottlenecks in the scalability of OpenFOAM on massively parallel clusters, Partnership for Advanced Computing in Europe (PRACE), white paper 2010, http://www.prace-ri.eu/IMG/pdf

[19] ESI-OPENCFD, OpenFOAM lid-driven cavity flow, http://www.openfoam.com/documentation /tutorialguide/tutorialse1.php

[20] N. Frasheri And E. Atanassov, Scalability Issues for Wind Simulation using OpenFOAM, Third Nesus Action Workshop NESUS 2016, IICT-BAS, Sofia, Bulgaria. October, 6-7, 2016.

[21] N. Frasheri And E. AtAnAssov, Scalability Issues for Wind Simulation Using OpenFOAM, Bulgarian Academy of Sciences, Journal Cybernetics and Information Technologies, 2017 (submitted).

Edited by: Aneta Karaivanova

Received: Dec 19, 2017

Accepted: Mar 27, 2018 